\title{
What complications are there that I should look out for?
}

\author{
Binny Kurkiakos, MD, MALCOLM C Champion, MB, FRCPC
}

\begin{abstract}
Comar OMPLICATIONS CAUSED BY INflammatory bowel disease (IBD) can be classified broadly into three subgroups (Table 1). The first subgroup consists of complications directly related to the activity or extent of the underlying intestinal disease. They may occur with acute exacerbations Of ulcerative colitis or Crohn's disease. The second subgroup, nutritional complications, are either due to an increased energy requirement of the patient or decreased nutrition intake because of symptoms related to eating. Finally, there are a variety of nonbowel or extraintestinal manifestations of IBD which may become symptomatic at the time of acute exacerbations of the underlying disease.
\end{abstract}

\section{COMPLICATIONS RELATED} TO BOWEL INFLAMMATION

Stricture: A stricture is a narrowing of a segment of bowel, either the small or large bowel. It may be more appropriate to consider a stricture as a clinical manifestation of Crohn's disease rather than a complication. In Crohn's disease, strictures can affect approximately $40 \%$ of patients and can be short or long, single or multiple (Figure 1). Strictures in Crohn's disease can be due to either acute inflammation or chronic fibrosis. Steroids can be effective in reducing acute inflammation but not improving strictures when there is chronic fibrosis. Small bowel strictures: These strictures are asymptomatic until they cause obstruction. Obstruction is defined as a slowing or stopping of the flow of bowel contents. Obstruction can be clinically subdivided into subacute (partial) or acute (complete) obstruction. Patients may present with abdominal pain, nausea and bloating, which normally occurs after eating and is exacerbated by large meals. The patient does not have any bowel movements or pass any flatus (gas). These symptoms may be relieved by vomiting. Passing a large amount of gas or liquid stool heralds relief of the episode of obstruction.

Became of the tendency of Crohn's disease to recur after surgery, surgical correction of the stricture is postponed until the patient is having significant symptoms of subacute obstruction, or the patient presents with complete bowel obstruction. Complete bowel obstruction requires surgery. A patient presenting with partial small bowel obstruction is treated with bowel rest, which may involve nasogastric aspiration of the gastric contents together with intravenous fluids and steroids. If they do not respond, surgery is required. In the 1990s there are two choices of surgery. The first is simple resection of the narrowed segment of bowel and reanastamosis of the two ends of the bowel together. The second is a stricturoplasty. A stricturoplasty involves dividing a narrowed portion of bowel and re-anastomosing it to remove the narrowing without removing any of the bowel. This type of surgery can be suc-

Ottawa Civic Hospital, Ottawa, Ontario

Correspondence and reprints: Dr MC Champion, 1053 Carling Avenue, Ottawa, Ontario KIY 4E9. Telephone (613) 761-4674

\begin{tabular}{l} 
TABLE 1 \\
Complications of inflammatory bowel \\
disease \\
\hline Bowel inflammation complications \\
Stricture \\
Massive hemorrhage \\
Toxic megacolon \\
Perforation/abscess \\
Fistulas \\
Carcinoma \\
Perianal disease \\
Nutritional complications \\
Anemia \\
$\quad$ iron deficiency \\
$\quad$ folate deficiency \\
$\quad$ B 12 deficiency \\
Malnutrition \\
Bone disease \\
Extraintestinal complications \\
Arthritis \\
Mouth lesions \\
Skin lesions \\
Eye lesions \\
Hepatobiliary complications \\
Thromboembolic problems \\
Kidney stones
\end{tabular}

cessful but, with time, restricturing can occur (Figure 2).

Large bowel strictures: Stricturing of the colon can be seen in both ulcerative colitis and Crohn's disease. In ulcerative colitis, its incidence has been reported to be as high as $11 \%$. It is more common in Crohn's disease because of the full thickness inflammation of the bowel wall. A colonic stricture in ulcerative colitis raises concern of carcinoma of the colon. Colonoscopy and biopsy of the stricture are necessary. However, this may not exclude an underlying carcinoma and it may be nec- 


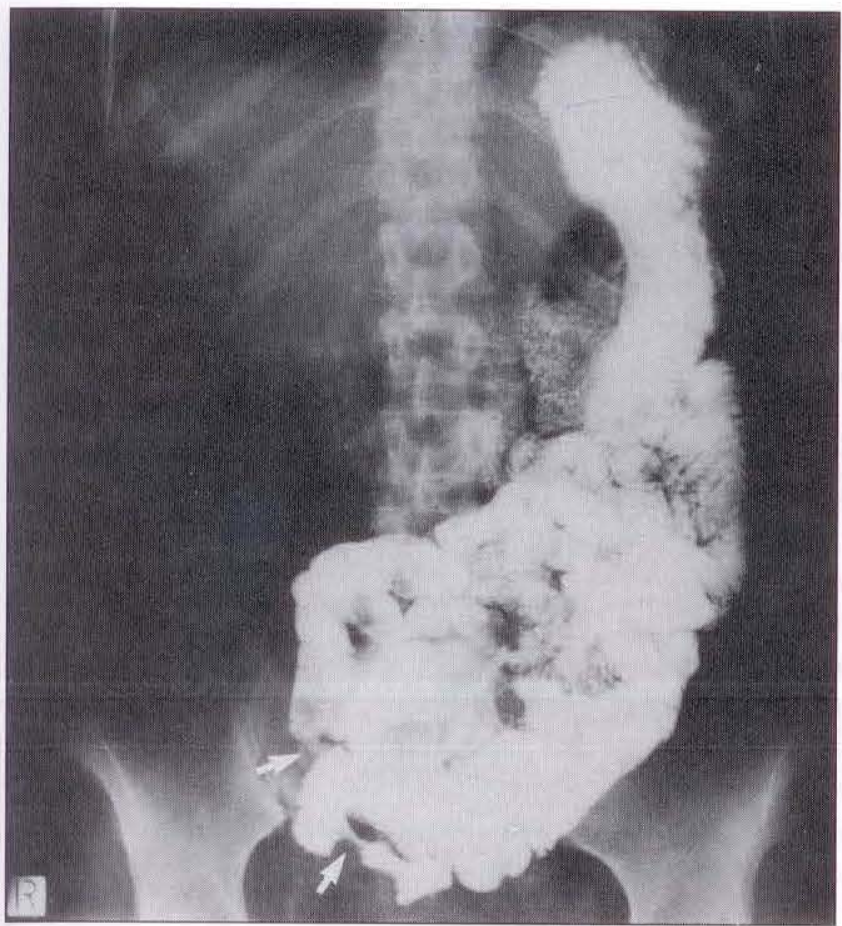

Figure 1) Small bowel stricture - antegrade small bowel enema in a patient with Crohn's disease. Arrow indicates a short stricture in the distal ileum

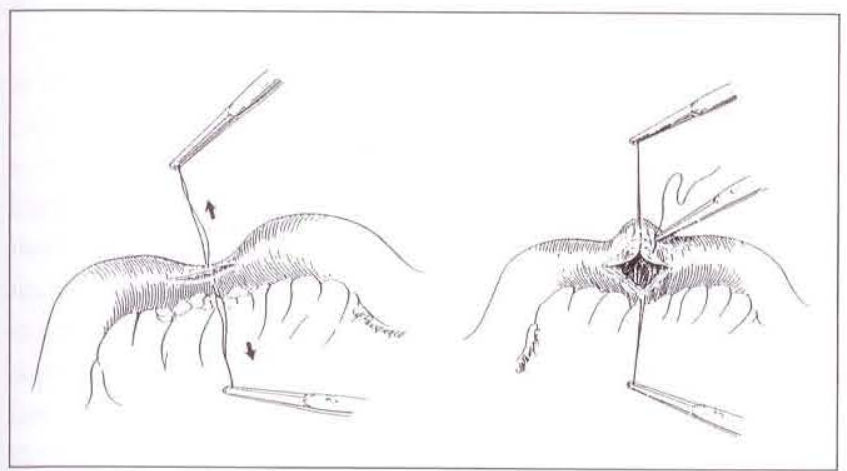

Figure 2) Stricturoplasty - after cutting through the stricture longitudinally (left), the opening is closed transwersely (right)

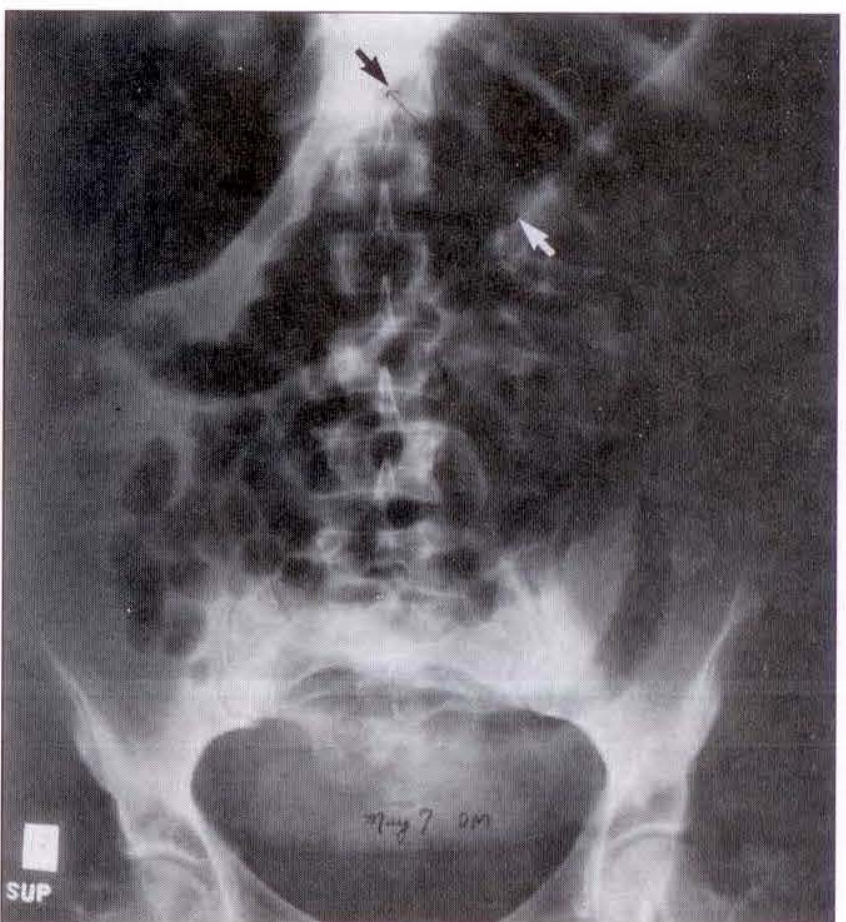

Figure 3) Toxic megacolom - arrows indicate dilated large bowel with a $14 \mathrm{~cm}$ diameter. Normal diameter of the large bowel is less than $6 \mathrm{~cm}$

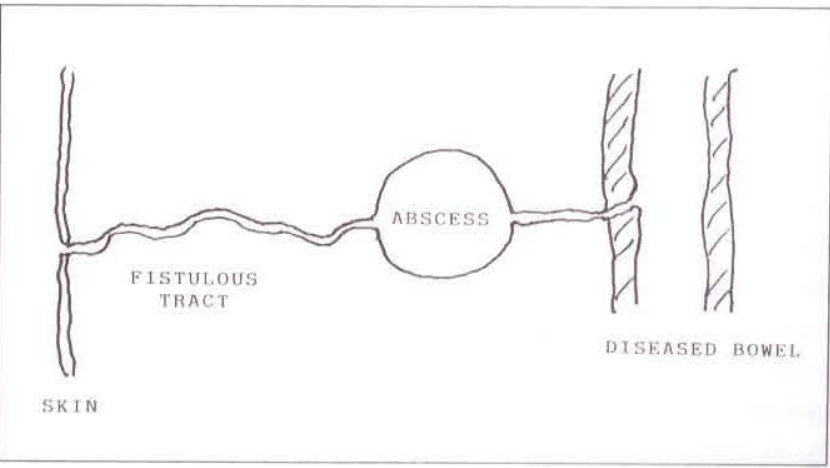

Figure 4) Abscess and fistula formation - intra-abdominal abscess arising from a small perforation. This has then drained onto the skin with a long fistulous tract essary to recommend colonic resection because of the possibility of a carcinoma within the bowel wall. In Crohn's disease, colonic strictures do not require surgery unless they become symptomatic. Most strictures are benign and do not cause symptoms. In fact, most are discovered incidentally on barium enema or colonoscopy. Colonoscopy is necessary to biopsy the stricture to exclude the rare cases of carcinoma in colonic Crohn's disease.

Unlike the small bowel, the colon is not amenable to stricturoplasty, and colonic resection is necessary. In some patients, balloon dilation of a short stricture is possible through a colonoscope.

\section{GASTROINTESTINAL BLEEDING}

Bloody bowel movements are a common manifestation of ulcerative colitis. They also occur with Crohn's disease but less commonly. Chronic bleeding can result in anemia which may require long term iron supplementation. Blood transfusions are rarely required, but are indicated when the hemoglobin is particularly low. A large gastrointestinal bleed is rare with ulcerative colitis or Crohn's disease. Rarely it can be presenting feature of small bowel Crohn's disease.

Most patients presenting with significant bleeding due to IBD will stop bleeding without requiring surgery. Initial management is intravenous fluids and blood transfusion, together with aggressive management of their inflammation.

Aggressive management of colitis and Crohn's disease include the use of intravenous steroids and aminosalicylic acid preparations. Surgery rarely is nec- 
essary to remove the area of localized bleeding in the small or large bowel with Crohn's disease. In patients with ulcerative colitis with a significant hemorrhage, a subtotal or total colectomy is indicated. Before surgery, the source of bleeding will need to be defined with endoscopy or an angiogram. An angiogram is an intra-arterial injection of dye followed by $\mathrm{x}$-rays which are taken to show dye leaking into the bowel from the blood vessel.

Toxic megacolon: Toxic megacolon occurs when the colon dilates and the patient simultaneously develops systemic toxicity with fairly rapid clinical deterioration (Figure 3). This normally is associated with an acute exacerbation of IBD and more commonly occurs with ulcerative colitis. Its incidence in the literature is reported to be as high as $2.5 \%$. However, with better management of the acute exacerbations of IBD, it is rare that one sees a case of toxic megacolon in the 1990s. In toxic megacolon, the affected segment of the bowel becomes intensely congested and the bowel wall becomes thin and necrotic. In ulcerative colitis this is due to ulcers and associated inflammation extending through the full thickness of the bowel wall. Although rare, toxic megacolon can occur in Crohn's disease. If it does occur, it usually is early in the course of the disease before the colon has become thickened and fibrotic. Several factors have been implicated in precipitating toxic megacolon. These are the use of narcotic painkillers, anticholinergic drugs, other drugs used to treat diarrhea and hypokalemia because of the diarrhea. A barium enema at acute inflammation has also been implicated in precipitating toxic megacolon.

Toxic megacolon appears to be more common in patients who have total colonic involvement. The clinical presentation is that of a severe attack of colitis with fever and a raised pulse. Blood tests normally show an increased white cell count and low albumin. Impending dilation often is heralded by a sudden decrease in the stool frequency because of decreased colonic evacuation. This frequently is associated with an increase in the amount of rectal bleeding, abdominal distention and tenderness. The most common complication of toxic megacolon is perforation of the bowel wall which results in shock and peritonitis due to leakage of the bowel contents into the abdominal cavity.

Treatment largely is preventative. Aggressive management of colitis with hospitalization of severe attacks has resulted in a significant decrease in the incidence of toxic megacolon. When it does occur, the patient requires aggressive rehydration and correction of any electrolyte abnormalities (particularly low potassium). Patients are also given intravenous steroids to decrease the inflammation and intravenous antibiotics to cover potential infections. The patient is followed closely by both the gastroenterologist and surgeon. The first $72 \mathrm{~h}$ are critical and if a patient does not respond to medical management, a subtotal colectomy with ileostomy is indicated. This aggressive management has resulted in a significant drop in mortality from $60 \%$ to between 5 and $10 \%$.

\section{PERFORATION CAUSING ABCESSES OR PERITONITIS}

Perforation occurs when a hole appears in the lining of the bowel and the bowel contents leak into the abdominal cavity causing inflammation of the peritoneum and peritonitis. Approximately $75 \%$ of perforations are associated with toxic megacolon. When it occurs in the absence of toxic megacolon, it usually is associated with an acute exacerbation of the IBD. The perforation occurs because of transmural (full thickness) inflammation of the bowel wall which results in a localized area breaking down and a hole forming. The resulting leakage can cause peritonitis or, in the case of small perforations, can become localized and form an abscess.

An abscess is a collection of pus and bowel contents in a walled-off or localized part of the abdominal cavity. It can be small ( 1 to $5 \mathrm{~mm}$ ) or large ( 10 to 15 $\mathrm{cm}$ ), single or multiple. Abscesses occur more commonly with Crohn's disease, but are rare in ulcerative colitis.

Perforation is suspected when a patient demonstrates a sudden deterioration with an increase in abdominal pain, fever and a raised white cell count. With generalized peritonitis, the patient is much more sick and toxic whereas with localized abscesses the patient may have less severe symptoms. On examination, the abdomen will be very tender and will demonstrate rebound tenderness (an increase in pain when the hand is taken away from palpating the abdomen). With an abscess this tenderness will be more localized and a mass may be palpable. These patients are often on steroids, which may mask the symptoms, but steroids have not been implicated in causing perforation. With peritonitis the tenderness and pain will be more generalized. The diagnosis of peritonitis can be confirmed by a plain abdominal $\mathrm{x}$-ray which may show the presence of free air in the abdominal cavity. Abscesses may be demonstrated on these plain abdominal $\mathrm{x}$-rays or alternatively by using ultrasound or a computed tomography (CT) scan of the abdomen. However, abscesses can be very difficult to demonstrate by radiological techniques.

The treatment for both peritonitis and abscesses is surgery. With peritonitis, the patient needs urgent surgery and, at the same time, will be rehydrated with intravenous fluids and started on intravenous antibiotics. With abscesses, localized drainage may be attempted but abdominal surgery more often is necessary. At surgery, the area of bowel involved is removed (a subtotal colectomy in ulcerative colitis and a localized resection in Crohn's disease).

Fistula: A fistula is an abdominal communication between any part of the gastrointestinal tract and either the body surface or another portion of the gastrointestinal tract or other organ. This means that fistulas can communicate with another part of the bowel (eg, ileal-colonic fistula), the skin (enterocutaneous fistula), the bladder (enterovesical fistula) and the vagina (enterovaginal fistula). Fistulas between different parts of the bowel are often asymptomatic but may present with diarrhea and pain. When a fistula opens either to the skin, bladder or vagina, there may be a small or large amount of feculent material draining from the fistula. Fistulas occur only with 

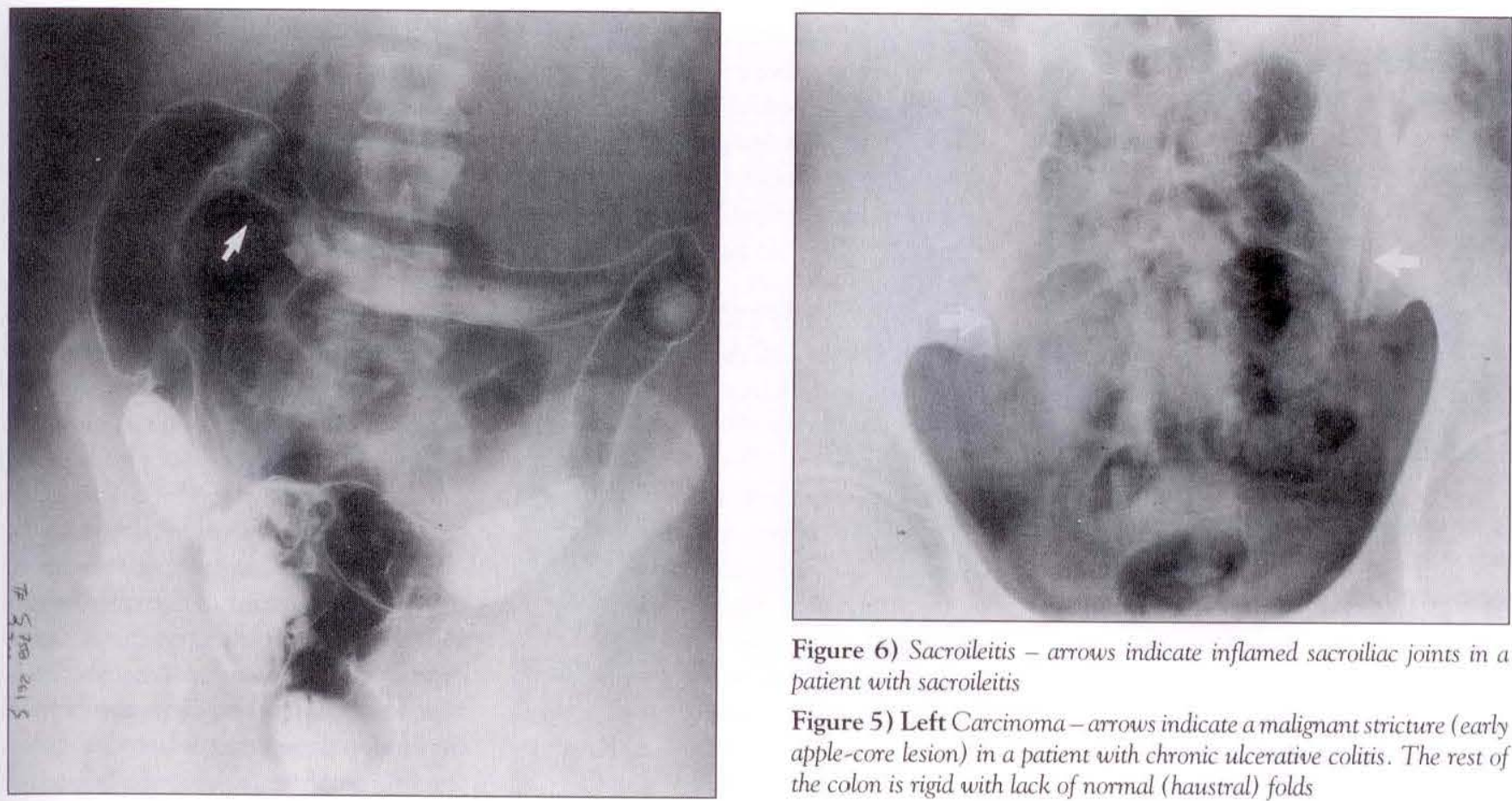

Figure 6) Sacroileitis - arrows indicate inflamed sacroiliac joints in a patient with sacroileitis

Figure 5) Left Carcinoma - arrows indicate a malignant stricture (early apple-core lesion) in a patient with chronic ulcerative colitis. The rest of the colon is rigid with lack of normal (haustral) folds

Crohn's disease. If a fistula occurs in a patient with ulcerative colitis, then the question has to be asked whether the patient has Crohn's colitis or ulcerative colitis.

A fistula often begins as an abscess that adheres to adjacent organs or the skin and then discharges its contents through a fistulous tract (Figure 4). This tract then becomes permanent. Fistulas can occur at any time but they are most often seen during the very active phases of the disease and particularly in the postoperative period after surgical resection.

Most fistulas eventually require surgery if they are causing significant symptoms. A small amount of discharge and no serious infections often is tolerated rather than subjecting the patient to major surgery. Medical management often is unsuccessful but includes bowel rest (often with total parenteral nutrition) and aggressive management of the underlying inflammation with steroids and aminosalicylic acid compounds. Some physicians may use 6-mercaptopurine which may be more beneficial than other medications in healing fistulas. Unfortunately, after having closed a fistula with medical therapy, the recurrence rate is at least $50 \%$ over the next year.
Carcinoma: Colonic carcinoma is probably the most serious complication of IBD, but fortunately it is rare (Figure 5). It tends to occur more frequently in ulcerative colitis than in Crohn's disease.

The risk of cancer in patients with ulcerative colitis has been shown to begin after seven years and then rises by approximately $10 \%$ every 10 years, reaching an approximate incidence of $35 \%$ at 30 years. A more recent study has demonstrated a cumulative 25 -year risk for patients with extensive colitis, with an intact colon, to be about $12 \%$.

The risk of colorectal cancer is not related to patient age or severity of bowel disease. However, patients whose colitis involves a greater portion of the bowel are at a higher risk of developing carcinoma. Patients with ulcerative proctitis do not have an increased risk of developing carcinoma.

The symptoms of carcinoma of the bowel are the same as for an exacerbation of colitis. Patients may complain of abdominal pain, a change in bowel pattern and may have rectal bleeding. On examination, an abdominal mass may be palpable. A patient with a carcinoma of the colon will not improve with conventional treatment for IBD. A diagnosis will be made either by barium enema or colonoscopy. Treatment is surgical, normally with a total colectomy.

There is a great deal of controversy in the literature regarding surveillance in patients who have longstanding ulcerative colitis to predict which patients are at risk of developing a cancer of the bowel. There is an association between dysplasia of the lining of the colon and the risk of cancer. Patients with marked dysplasia are at a definite risk of developing cancer of the colon. For this reason colonoscopy with multiple biopsies of involved bowel has been recommended on a regular basis. It is recommended that colonoscopic surveillance should be performed every one to two years after eight years of total colitis and after 13 years of left-sided colitis. If the biopsies do not show any evidence of dysplasia, then the interval between surveillance colonoscopy can be increased according to the individual gastroenterologist's interpretation of the literature. Many experts believe that the risk of carcinoma in ulcerative colitis has been exaggerated and that colonoscopies are being performed too early and too frequently in patients with longstanding ulcerative colitis. It is therefore expected that the recommendations for surveillance colonoscopy will change over the next few years. 


\section{TABLE 2}

\section{Extraintestinal manifestations of in- flammatory bowel disease}

\begin{tabular}{lc}
\hline Related to disease activity & $\begin{array}{c}\text { Frequency } \\
(\%)\end{array}$ \\
\hline $\begin{array}{lc}\text { Mouth and skin problems } \\
\text { Aphthous ulceration }\end{array}$ & 20 \\
Erythema nodosum & 2 to 10 \\
Pyoderma gangrenosum & 0.5 \\
Acute arthritis & 6 to 12 \\
Eye complications & \\
Conjuctivitis & 4 to 10 \\
Episcleritis & \\
Uveitis & \\
Unrelated to disease activity & \\
Sacroileitis & 18 \\
Ankylosing spondylitis & 2 to 6 \\
\hline
\end{tabular}

Perianal disease: Anal fissures, abscesses and fistulas are uncommon complications of ulcerative colitis but occur in as many as $82 \%$ of patients with Crohn's disease.

An anal fissure is a linear ulcer within the anal canal. Patients will complain of pain when defecating and may have a small amount of bleeding. Treatment is to avoid constipation together with local therapy with suppositories or creams normally containing a small amount of steroids.

A perianal abscess usually presents during exacerbation of disease. A patient will experience pain when defecating and this pain often is aggravated by sitting. The patient may complain of a hard, tender lump in the anal region. The abscess will drain spontaneously either into the rectum or on to the skin. When this happens a fistula-in-ano can occur in which there is a separate fistulous tract bypassing the anus between the rectum and the skin. The patient may notice a small or large amount of discharge from the fistulous opening which is normally near the anus.

Perianal abscesses need aggressive treatment with antibiotics as soon as they are noticed. Early treatment reduces the risk of fistulous development. Different antibiotics can be used but metronidazole (Flagyl; Rhône-Poulenc) has been shown to be particularly effective in perianal Crohn's disease. Some fistulas will close spontaneously whereas others will close with the use of antibiotics. Recurrent fistulas unre- sponsive to medical treatment may need surgery. However, surgery is difficult and a successful outcome is not guaranteed. This may mean that the patient has to accept a small amount of discharge rather than undergo extensive surgery or even a colectomy.

\section{NUTRITIONAL COMPLICATIONS}

Nutritional deficiencies are more common in Crohn's disease than in ulcerative colitis. This is because in Crohn's disease more of the small bowel is involved; therefore, symptoms are often brought on by eating and absorption of nutrients. Malnutrition occurs from a combination of poor appetite, avoiding food because eating produces symptoms, and the loss of protein from the bowel and through fistulas. Patients with active inflammation also have a higher energy requirement because of their disease. The most common nutritional complication of IBD is anemia due to iron deficiency. The iron deficiency is due to chronic blood loss, in quantities too small for the patient to notice. In addition, poor dietary intake and decreased absorption of iron may exacerbate this deficiency.

Involvement of the different parts of the bowel can result in specific types of malabsorption. Jejunal involvement can result in malabsorption of folate or iron, both of which can contribute to the patient's anemia. Crohn's disease of the terminal ileum can result in vitamin B12 malabsorption and deficiency of bile salts; for this to occur, at least $90 \mathrm{~cm}$ of ileum must be diseased or removed. With bile salt deficiency, fat absorption also is impaired, resulting in decreased absorption of fat-soluble vitamins (vitamins A, D, E and K). Malabsorption of vitamin $\mathrm{D}$ can result in bone disease (osteoporosis and/or osteomalacia). Finally, in patients with diarrhea, there is a number of electrolytes which can become depleted, particularly potassium.

The treatment of nutritional complications related to IBD largely is preventative. A healthy balanced diet is important in all patients. Some physicians may prescribe a high fibre diet whereas patients with small bowel strictures will need a low residue diet. In the patient with mild Crohn's disease or Crohn's disease in remission, there does not seem to be any specific recommendations for a diet. Patients should avoid foods which exacerbate their symptoms, but rigid elimination diets are controversial. Some patients will have acquired lactose intolerance and benefit from avoiding milk and milk products.

Patients with particular nutritional deficiencies will require replacement therapy, such as iron, folate, vitamin D or calcium oral supplementation. Vitamin $B_{12}$ has to be given by an intramuscular injection, normally once a month.

Patients with significant malnutrition require caloric supplementation, initially using one of the many liquid enteral feedings available on the market. These enteral feedings can be subdivided into elemental and defined formula preparations. Normally $1 \mathrm{~mL}$ of these supplements contains $4.187 \mathrm{~J}$ and this means a large number of calories can be given in a small volume of liquid. The defined formula preparations are high in calories but require some digestion prior to absorption. The elemental preparations do not require digestion as the fat, protein and carbohydrates are already in their digested form and are ready for absorption. In Crohn's disease aggressive nutritional therapy during an attack may have a primary therapeutic role as well. Bowel rest (ie, through total parenteral nutrition) probably is unnecessary and patients can be treated with an elemental diet as part of their medical management. This can be administered via continuous infusions through a small nasogastric tube if the patient cannot tolerate oral feedings. Alternatively, the newer elemental diets are more palatable and can be taken orally. Intravenous nutritional support (total parenteral nutrition) may be necessary for the severely ill and nutritionally depleted patient, in those with fistulas or in Crohn's disease patients who have lost a large amount of small bowel function to disease or resection.

\section{EXTRAINTESTINAL MANIFESTATIONS}

Extraintestinal manifestations, such as arthritis, skin rashes, inflammation of the eye, gallstones and liver bile duct 
disorders, occur in one-quarter to onethird of patients with either ulcerative colitis or Crohn's disease (Table 1). Although Crohn's disease and ulcerative colitis differ in the way they affect the bowel, they are quite similar in the kind of systemic complications they cause, and the incidence is about the same for both diseases. In most patients these complications are relatively mild and more of a nuisance than anything else; however, in approximately $1 \%$ of patients, they cause major difficulties.

Some manifestations, including arthritis, erythema nodosum and eye inflammation, occur mainly when the bowel is actively inflamed (Table 2). One can usually predict that these specific problems will be controlled or eliminated when the intestinal disease is suppressed by medical therapy or when the entire colon is removed from the patient with ulcerative colitis. Sometimes these symptoms are an early sign of an exacerbation of a patient's IBD.

Certain types of chronic extraintestinal problems, such as ankylosing spondylitis and sclerosing cholangitis, are probably related to the bowel disease, but their activity may continue long after the colitis is quiescent or a colectomy has been performed.

Other extraintestinal manifestations of Crohn's disease, such as gallstones and kidney stones, often are due to an inadequate or disordered intestinal function, with resultant effects on other organs.

Arthritis: Arthritis is the most common extraintestinal manifestation in both Crohn's disease and ulcerative colitis, with an incidence of 4 to $23 \%$. It is possibly more common in Crohn's disease. The arthritis may effect the peripheral joints or the lower spine.

Peripheral arthritis may present with painful, red, swollen joints, most commonly the hips, ankles, wrists and elbows. Small joints are rarely involved. The arthritis only affects the lining of the joint cavity (synovium) and there is no erosive damage which often is seen in patients with rheumatoid arthritis. A flare-up of arthritis usually occurs during an exacerbation of the IBD and will respond to medical therapy for the IBD.

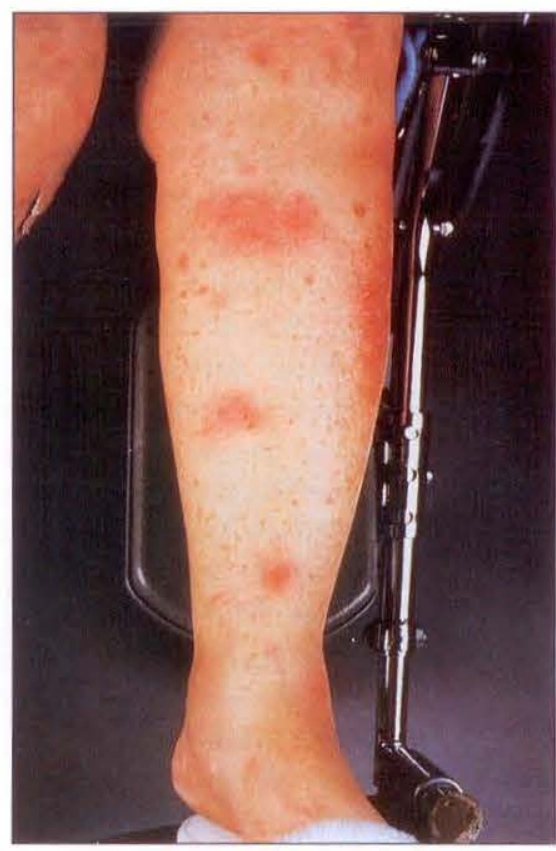

Figure 7) Erythema nodosum - patient with erythema nodosum associated with acute flareup of Crohn's disease. Several small red nodules on the anterior aspect of the legs are seen

Steroids have a strong anti-inflammatory action and are used in the treatment of other causes of arthritis. Sulphasalazine (Salozopyrine; Pharmacia) also is effective in treating arthritis, and was originally first designed as an anti-arthritis medication. Together with physiotherapy, patients often improve and do not require nonsteroidal anti-inflammatory drugs (NSAIDs). NSAIDs are particularly effective in treating arthritis but unfortunately some NSAIDs have been implicated in exacerbating IBD. For this reason, NSAIDs should only be used with the careful supervision of the patient's physician. Sometimes the joint complications recur when patients with bowel disease are being withdrawn from prednisone therapy. This can be particularly frustrating for the patient who is trying to get off long term corticosteroids, and it is in these patients that NSAIDs may be helpful.

Ankylosing spondylitis is an inflammation of the spine which presents with pain and stiffness of the spinal column. It occurs in 3 to $6 \%$ of patients with IBD and it is equally frequent in Crohn's disease and ulcerative colitis. The pain and lower back stiffness is worse in the

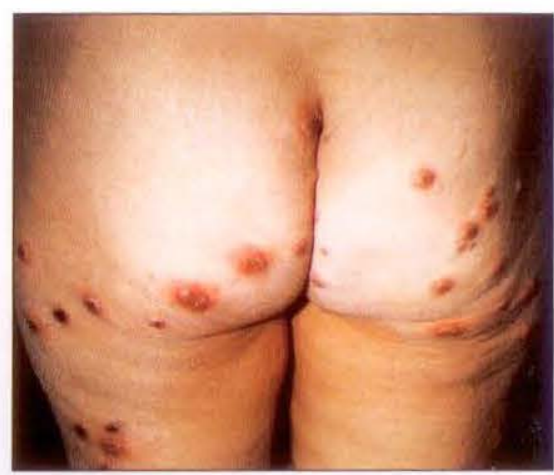

Figure 8) Pyoderma gangrenosum - small bullous-like ulceration in a patient with ulcerative colitis

morning and is relieved by exercise and physiotherapy. The symptoms are worsened by inactivity and rest. This disorder usually is associated with a specific tissue type (HLA B27). Treatment is difficult and improvement is not related to improvement in the activity of the bowel disease.

Sacroileitis is an inflammation of the joints between the lower back (sacrum) and the pelvic bone (iliac). It often is asymptomatic and may be found on routine $\mathrm{x}$-rays. A high proportion of these patients will have coexisting spondylitis (inflammation of the spine). The main symptom of sacroileitis is a low back pain which is worse in the morning and relieved by exercise (Figure 6).

The mainstay of treatment for ankylosing spondylitis and sacroileitis is regular physiotherapy and exercises to maintain the mobility and flexibility of the spinal column. Together with simple analgesics, such as acetaminophen (Tylenol; McNeil), patients often do not require the stronger NSAIDs.

Mouth lesions: Aphthous stomatitis often occurs in the mouth at the same time as an exacerbation of IBD. It can occur in up to $20 \%$ of patients with IBD and is more common in patients with Crohn's disease. Most episodes of aphthous ulceration are self-limiting and last three to five days regardless of the treatment of the underlying bowel disease. More painful lesions sometimes improve with local therapy using steroids (prednisone solution) and local anesthetic cream.

On rare occasions Crohn's disease can involve the lips, gums and buccal 

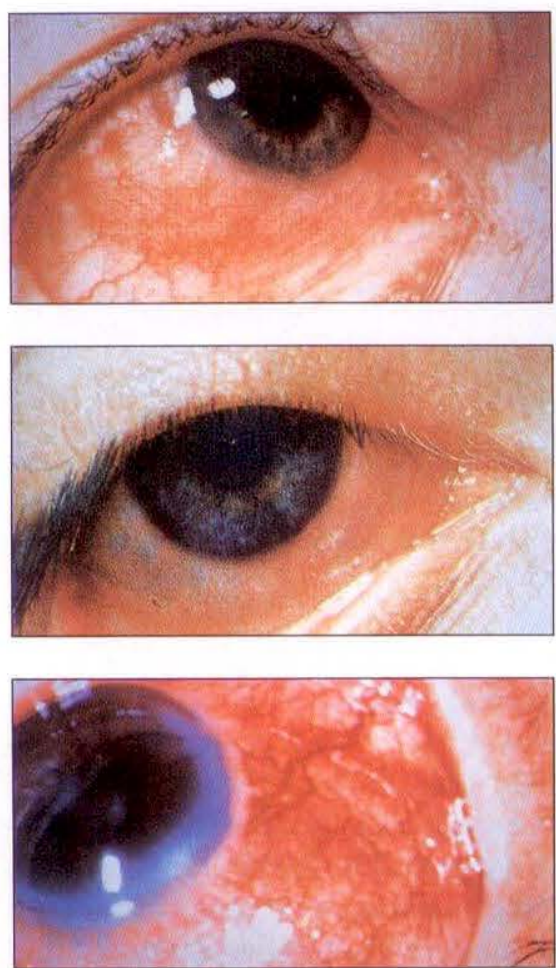

Figure 9) Eye complications of inflammatory bowel disease. Top Conjunctivitis; Middle Episcleritis; Bottom Uveitis

mucosa. It always is associated with intestinal involvement and responds to therapy for intestinal Crohn's disease.

Skin rashes: Erythema nodosum occurs in approximately 2 to $10 \%$ of patients with IBD. This condition appears to be more common in women and consists of painful red nodules which usually occur below the knee on the shins but can occur on the upper legs (Figure 7). It often is associated with an exacerbation of the IBD and subsides as the inflammation improves. Diagnosis is made clinically but a skin biopsy rarely is necessary. It is most common with Crohn's disease of the colon and most patients have had an episode of arthritis some time during the course of their disease.

Therapy is directed at treating the bowel inflammation. This may involve medical or surgical treatment. Some dermatologists may use potassium iodine applied topically to the lesion, or alternatively prescribe an NSAID to be taken orally.

Pyoderma gangrenosum starts with a raised, red, tender area normally on the lower leg, either on the shin or ankle. This lesion then progresses into a boil or bullous-like lesion which breaks down resulting in ulceration (Figure 8). This ulceration can be quite extensive and can result in widespread tissue skin damage and necrosis. Pyoderma gangrenosum has an overall incidence of 0 to $5 \%$ and occurs more commonly in ulcerative colitis than in Crohn's disease patients. Most patients (85\%) have pancolitis, and persistent and extensive pyoderma gangrenosum is a very rare indication for a colectomy.

Steroids have been shown both to halt progression of the disease and to prevent development of new lesions. Sometimes even higher doses of prednisone are used for pyoderma gangrenosum than is normal in the treatment of IBD. Other drugs which can be used include: sulphasalazine, dapsone (Avulosulfon; Ayerst) and, more recently, chlofazamine.

Eye problems: There are a variety of eye or ocular lesions which can occur in patients with IBD. It is difficult to ascertain the true incidence as many patients do not have routine eye examinations and these lesions can be asymptomatic. The incidence varies between 4 and $10 \%$, with most common conditions being conjunctivitis, episcleritis or uveitis. Other frequent complications includes cataracts, ulceration of the cornea and retrobulbar neuritis.

The mechanism for eye problems related to IBD is unknown but there is a definite association between eye lesions and exacerbations of IBD. Eye problems with IBD are often associated with other nonsystemic manifestations such as arthritis and erythema nodosum.

Treatment for eye complications (Figure 9) includes treating the underlying active bowel disease as well as the use of hydrocortisone solution in the eye. Serious visual loss can occur with some forms of uveitis, requiring expert ophthalmological care.

Hepatobiliary problems: There are a number of liver and bile duct complications found in patients with IBD.

Steatosis occurs when there is an increased amount of fat deposited into the liver. It may present with an enlarged liver or abnormal liver function blood tests. Its true incidence is unknown, but it is thought to occur com- monly in patients who have active inflammation of the bowel. The cause is unknown but it possibly is secondary to malnutrition and protein depletion due to the active disease.

Treatment is the same as for the underlying bowel disease. Steatosis has not been demonstrated to progress to more serious liver disease such as cirrhosis.

Pericholangitis is a condition in which the small bile ducts in the liver become edematous and filled with inflammatory cells. It occurs in approximately $5 \%$ of the patients with IBD. In patients who have had liver biopsies performed because of abnormal liver function blood tests, about one-third have this bile duct problem. This is usually in association with inflammation of the bile ducts outside the liver. Pericholangitis is normally asymptomatic but may present with abnormal liver function blood tests or an enlarged liver. It occurs more frequently in patients with extensive bowel disease, particularly colitis. Evaluation of the patient with pericholangitis on liver biopsy should include an $\mathrm{x}$-ray of the bile ducts, normally performed by endoscopic retrograde cholangiographic pancreatography (ERCP) to rule out large duct disease, namely sclerosing cholangitis.

Sclerosing cholangitis is inflammation of the large bile ducts, both within the liver and in the biliary system. It is a progressive disease which results in fibrosis and stricturing of the bile ducts. It occurs in up to $4 \%$ of patients with IBD and is more common with colitis of either etiology. It is rare with small bowel Crohn's disease. It may progress even after the colon has been removed surgically. This disease usually affects males and there is no relationship to the duration or activity of the colitis. It presents with jaundice, itching and abnormal blood tests. There is evidence of marked elevation of some of the liver function blood tests and ERCP examination demonstrates stricturing and fibrosis of the bile ducts, both outside and within the liver.

There is no definitive curative treatment for pericholangitis or sclerosing cholangitis. Some patients may benefit temporarily from drainage of the bile ducts, with a synthetic tube (stent) be- 
ing placed inside the ducts relieve an area of blockage. This can normally be achieved endoscopically. Other patients with very severe sclerosing cholangitis go on to receive liver transplants.

Cholangiocarcinoma (cancer of the bile ducts) occurs 10 times more frequently in patients with IBD than in the general population. It occurs primarily in patients with ulcerative colitis and rarely is seen in patients with Crohn's disease. Despite this increased incidence, it is a rare complication of IBD. It is associated with sclerosing cholangitis and patients may complain of weight loss, progressive jaundice and right upper quadrant pain. Surgical resection rarely is successful and patients are usually managed palliatively with insertion of stents endoscopically or surgically. Unfortunately liver transplantation has not improved the survival of these patients.

Gallstones occur in up to $30 \%$ of patients with Crohn's disease of the terminal ileum. This results from decreased bile salt absorption in the distal ileum and subsequent decrease in the amount of bile salts available to allow cholesterol to dissolve in the bile. This imbalance of insoluble cholesterol leads to the formation of gallstones. Patients with symptomatic gallstones require a cholecystectomy. Asymptomatic gallstones discovered on routine ultrasound examination or other $\mathrm{x}$-rays do not require surgery.

Thromboembolic disease: The incidence of thromboembolic complications in IBD has been quoted as high as

ACKNOWLEDGEMENTS: Dr Champion gratefully acknowledges $\mathrm{Dr} \mathrm{H}$ Tao, Department of Radiology, Ottawa Civic Hospital, for supplying the $\mathrm{x}$-rays.

\section{SUGGESTED READING}

1. Sidorov J. Inflammatory Bowel

Disease, Diagnosis and Complications, vol 19, series 4. Montreal: Medicine North America, 1991.

2. Cello JP. Ulcerative colitis. Donaldson RM Jr, Crohn's disease. In: Sleisenger $\mathrm{MH}$, Fordtran JS, eds. Gastrointestinal Disease. Pathophysiology, Diagnosis, Management, 3rd edn. Philadelphia: WB Saunders, 1989:1122-68, 1089-122.
$6 \%$, but overall it appears to be a rare complication. The risk of increased thrombosis is thought to be due to an increase in some of the clotting factors and platelets which are found in some patients with IBD. This tendency for increased thrombosis means IBD patients should use the oral contraceptive pill with caution as it has also been implicated in causing thromboembolic disease.

Treatment largely is preventative, with early mobilization after surgery and regular leg exercises if the patient is on prolonged bed rest. Should a deep venous thrombosis develop in the legs, the patient should be given anticoagulants to thin the blood, initially intravenous heparin and subsequently warfarin (Coumarin; DuPont) for up to one year. Kidney stones: The incidence of kidney stones is two to 10 times higher in patients with IBD. In some patients this is due to uric acid crystals caused by increase cell turnover in patients with chronic inflammation. In others it is due to calcium oxalate or calcium phosphate making up the bulk of the stones.

Kidney stones composed of oxalate crystals are more common in patients with Crohn's disease of the ileum, usually after one or two bowel resections. The mechanism for the formation of these oxalate kidney stones also involves bile salts. When the amount of bile salts available for promoting fat absorption is too low, excessive amounts of dietary fat remain unabsorbed in the intestine. The malabsorbed fat binds calcium from the diet and

3. Complications of ulcerative colitis, Complications of Crohn's disease. In: Thompson WG, ed. The Angry Gut. Coping with Colitis and Crohn's Disease. New York: Plenum Press, 1993:97-113,139-64.

4. Allan RN. Extra-intestinal manifestations of inflammatory bowel disease. Clin Gastroenterol 1983;12:914.

5. Rankin GB, Watts HD, Melnyk IS, et al. National Cooperative Crohn's Disease Study: Extra-intestinal manifestations and perianal complications. Gastroenterology 1979;77:914.

6. Gravelle EM, Kantrowitz FG. Arthritic manifestations of inflammatory bowel from bodily secretions. Normally this calcium would bind with oxalate in the diet and carry them out in the feces. Oxalates which are not bound by calcium are absorbed and excreted in the urine. When there are large quantities of oxalates in the urine it can precipitate and form kidney stones.

Most kidney stones will pass spontaneously and surgery rarely is required. At present, lithotripsy can be used to break up the kidney stones using high energy sound waves. Treatment also includes reducing the dietary intake of oxalate-containing foods, including tea, cola, chocolate, cocoa and vegetables with a high oxalate content. If the patient has malabsorption of fat, lowering the fat intake may be helpful in decreasing the amount of oxalate in the urine. A high urine output is also important, facilitated by ingesting large quantities of fluid daily.

\section{CONCLUSIONS}

There are many complications of IBD. Fortunately, most patients will experience only one or two of these complications, and some will not experience any. A few patients, however, will have multiple complications. The first approach to complications of IBD is an understanding and awareness of potential problems. Patients must rely on the expertise of their gastroenterologist in treating these complications. When necessary, further input may be required from specialists in surgery, rheumatology, dermatology or ophthalmology.

disease. Am J Gastroenterol 1988;83:703.

7. Shoetz DJ Jr, Coiler JA, Verdinheimer MC. Pyoderma gangrenosum and Crohn's disease. Eight cases and review of the literature. Dis Colon Rectum 1983;26:155.

8. Knox DL, Schaehat AP, Mustonen E. Primary, secondary and coincidental ocular complications of Crohn's disease. Ophthalmology 1984:91:163.

9. Vierling JM. Hepatobiliary complications of ulcerative colitis and Crohn's disease. In: Zakim D, Buyer ED, eds. Hepatology: A Text Book of Liver Disease. Philadelphia: WB Saunders, 1987:385-96. 


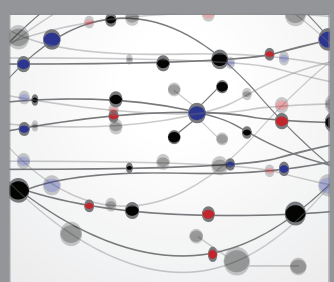

The Scientific World Journal
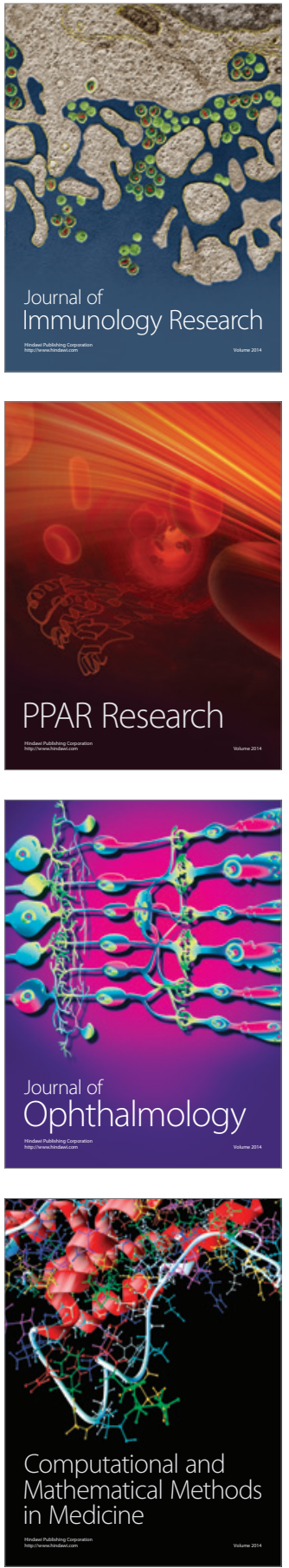

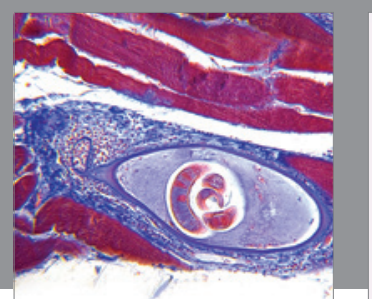

Gastroenterology Research and Practice

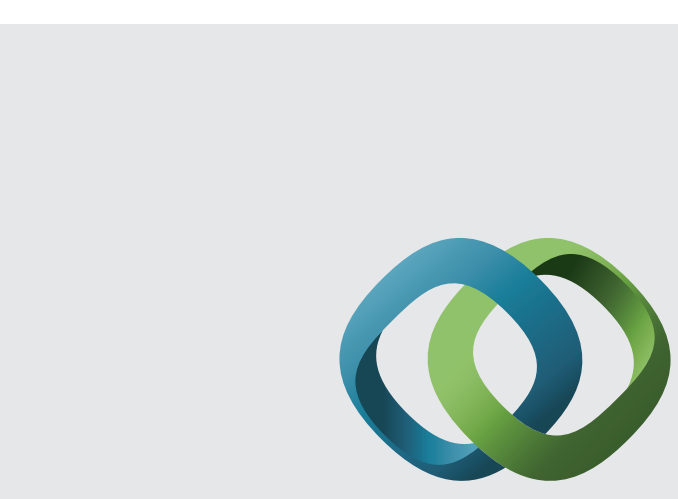

\section{Hindawi}

Submit your manuscripts at

http://www.hindawi.com
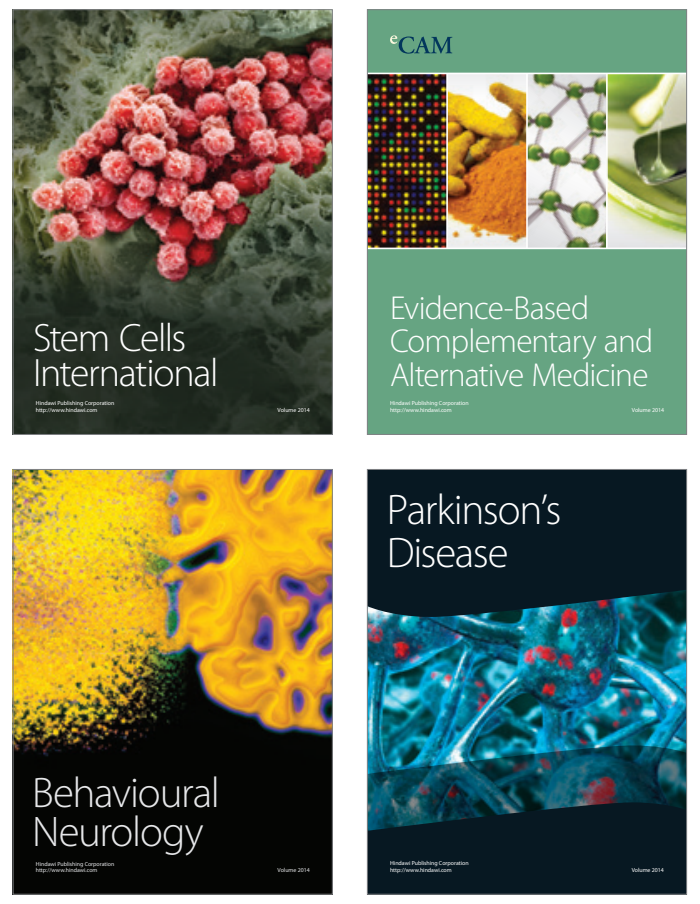
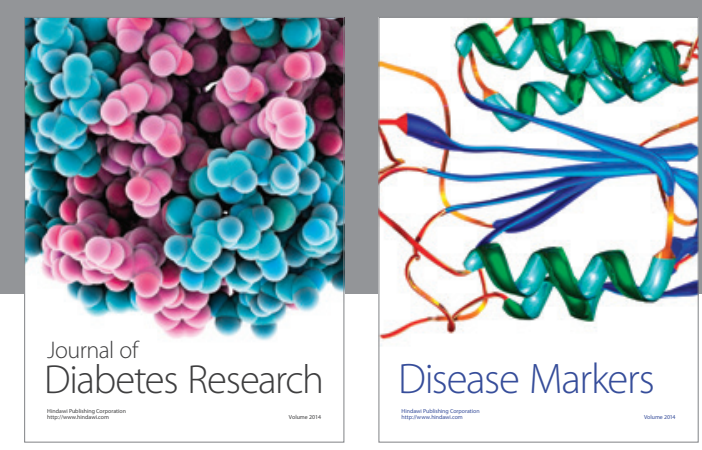

Disease Markers
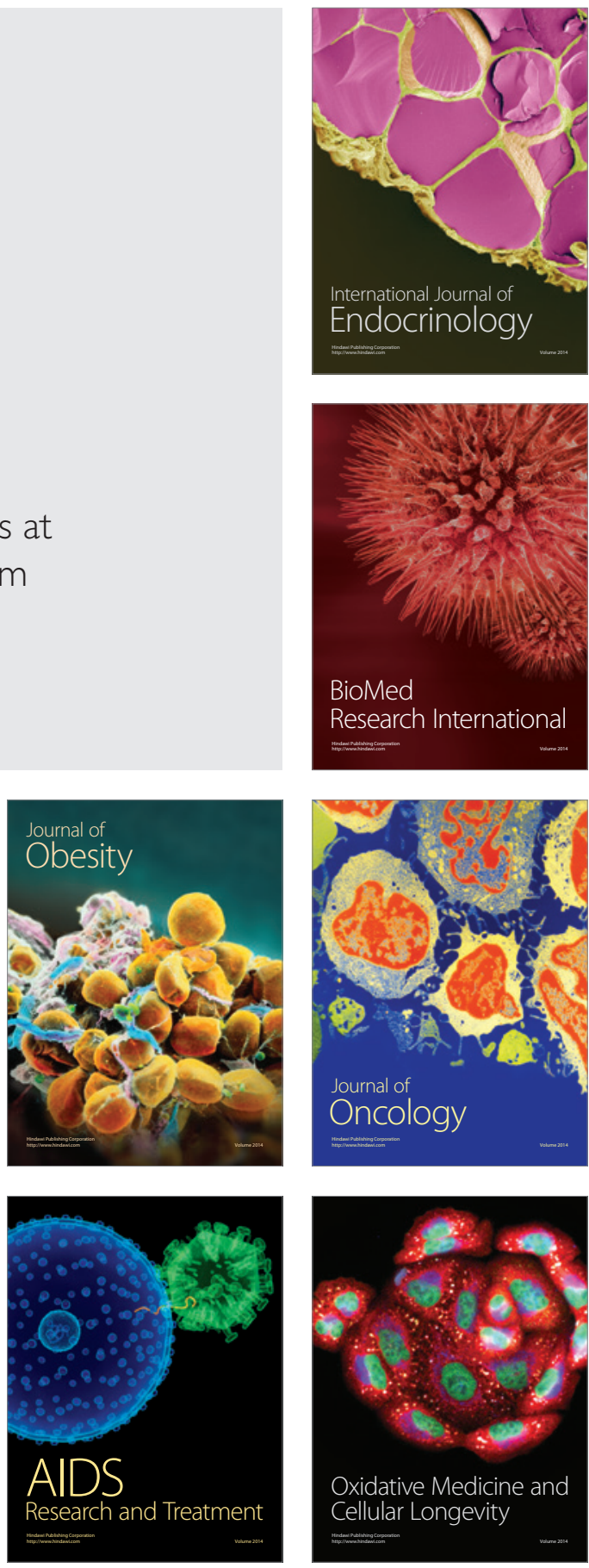